\title{
Development of the Rheumatoid Arthritis Distress Scale (RADS) - A New Tool to Identify Disease- Specific Distress in Patients with Rheumatoid Arthritis
}

\author{
Linda Silke ( $\square$ Isilke@tcd.ie ) \\ King's College London https://orcid.org/0000-0003-4281-9690 \\ Othman Kirresh \\ Chelsea and Westminster Hospital \\ Jackie Sturt \\ King's College London \\ Heidi Lempp \\ King's College London
}

\section{Research article}

Keywords: Distress, patient reported outcome measure, qualitative research, Rheumatoid Arthritis, secondary analysis

Posted Date: February 1st, 2021

DOl: https://doi.org/10.21203/rs.3.rs-183853/v1

License: (9) This work is licensed under a Creative Commons Attribution 4.0 International License. Read Full License

Version of Record: A version of this preprint was published at BMC Rheumatology on November 16th, 2021. See the published version at https://doi.org/10.1186/s41927-021-00220-4. 


\section{Abstract}

Background. Patients with Rheumatoid Arthritis (RA) may experience psychological distress (depression, anxiety) in addition to their physical symptoms. People with RA may also experience disease-specific distress (DSD), related to the specific burden of living with their life-long condition. DSD is a patient reported outcome in several long-term conditions, including type 1 and 2 diabetes. The aims of this study were to determine whether DSD is experienced by people with RA, and if so, develop a Patient Reported Outcome Measure (PROM) to assess for DSD in people with RA.

Methods. A five-phased qualitative study was conducted which consisted of a secondary data analysis of 61 interviews of people with rheumatological disease (Phase 1), validation of findings via a Patient and Public Involvement (PPI) group of people with RA ( $n=4)$ (Phase 2), item generation for a PROM (Phase 3) and establishing face and content validity of the PROM via PPI group $(n=4)$ and individual cognitive interviews ( $n=9)$ of people with RA respectively (Phase 4 and 5). The final PROM was presented at a Patient Education Evening for patients with long-term rheumatological conditions, including RA, and carers.

Results. Five themes of rheumatological disease distress emerged from Phase 1, which were validated in the Phase 2 PPI group. After Phases 3-5, the Rheumatoid Arthritis Distress Scale (RADS) was formed of 39 items and 3 supplementary questions. Overall participants reported the content of the RADS to be clear and relevant, and that DSD is a valid concept in RA, distinct from other entities like clinical depression or anxiety.

Conclusions. DSD appears to be an important concept in RA. The 39-item RADS demonstrates acceptable face and content validity in this patient group. Further psychometric testing is needed. The RADS may be a useful tool for healthcare professionals to identify RA distress.

\section{Background}

Rheumatoid arthritis (RA) is a progressive inflammatory disease, which causes pain, joint damage and disability, and affects $0.5-1 \%$ of the adult population (1). Patients with RA commonly experience psychological distress in addition to their physical symptoms, and increased incidences of depression and anxiety have been reported in RA populations (2-4).

Disease-specific distress (DSD) is one form of psychological distress, which has been identified in several different long-term conditions such as cancer (5-7), type 1 and 2 diabetes (8), and Inflammatory Bowel Disease (IBD) (9). DSD refers to the distress or burden of living with a particular long-term condition, its symptoms and/or treatments. People with DSD exhibit signs of subjective stress that are not necessarily consistent with those of a diagnosable mental health condition (10). This means that patients can have DSD without being diagnosed with depression or anxiety $(6,9,11)$. 
In diabetes, where the evidence is most established, higher levels of DSD, not depressive symptoms or clinical depression, are associated with out of target blood glucose levels (12), and interventions that effectively reduce diabetes distress can improve patients' glycaemic control $(13,14)$.

DSD in long-term rheumatological conditions has not yet been described in the literature. Given the progress in the detection and management of diabetes distress, and the positive clinical outcomes following effective interventions $(12,13)$, this current study is important for patients who experience distress when diagnosed with RA, and for their clinicians to offer evidence-based based treatment. The development of a Patient Reported Outcome Measure (PROM) to identify DSD in people with RA seems therefore timely as a first step towards comprehensive care and management.

The aims of this study were to 1 . determine if there is any evidence of DSD in patients with RA and 2. if so, to develop a PROM to identify DSD in people with RA. The specific objectives were to:

- re-analyse existing interview transcripts and identify indicators of DSD in RA and other rheumatological diseases; Antiphospholipid Syndrome (APS) and Idiopathic Inflammatory Myopathy (IIM)

- validate the secondary qualitative data prospectively with patients who attend a rheumatology outpatient clinic at a local teaching hospital

- generate items for a PROM through themes of distress identified from the secondary data anaysis

- establish face and content validity of the PROM and adjust items as required, through a Patient and Public Involvement (PPI) group, individual cognitive interviews and during an education evening with people with RA.

\section{Methods}

\section{Study Design}

This was a five-phased qualitative research study, which aimed to follow previous research methods in developing PROM's (15-19) and use criteria for reporting qualitative research (20).

In Phase 1, a secondary thematic qualitative data analysis was undertaken retrospectively from 61 audiorecorded 1:1 interview transcripts from four existing data sets, with interview studies dating between 2004-2015 (21-24). Primary research questions focused on the impact of fatigue and inactivity in IIM and APS, and the experiences, expectations and needs of patients with RA about their disease management (21-24). In Phase 2, a PPI group of people with RA $(n=4)$ was set up to explore and validate the findings of the thematic analysis. In Phase 3 , items for a PROM were generated from the identified themes of rheumatological disease distress. In Phase 4, a PPI group of people with RA $(n=3)$ was consulted with the aim of establishing face and content validity of the measure and performing initial item reduction. In Phase 5, the PROM was presented to people with RA and individual cognitive interviews $(n=9)$ were conducted to further establish face and content validity, refine items where 
necessary and ensure the PROM 'made sense' to participants. The final draft was presented at a Patient Education Evening for patients with long-term rheumatological conditions, including RA, and carers.

\section{Ethical Considerations}

All four anonymized original data sets used in Phases 1-3 had received written ethics approval (21-24). Author H.L. was an investigator in the primary research for all four studies. Following review of the original protocols, it was determined that the aims of the secondary analysis were closely aligned with the original studies' overall aims for which written consent was previously obtained.

The local University Ethics Committee provided ethics approval for Phases 3-5 on 24.04.2018 (REC Number: MRS-17/18-6443). Participants undertook informed consent procedures. Cognitive interviews were transcribed, following removal of identifiable data, by L.S. and a professional transcription service. All audio files were deleted following transcription. All face-to-face interviews and PPI groups were held in a private room in a Medical School.

\section{Study Sample}

The views and perspectives of a total of 77 people with rheumatological disease were involved in phases 1-5 with specific sample detail presented in Fig. 1.

Phase 1 demographic data was previously reported (21-24) and was not formally collected during Phases 2-5.

We aimed to recruit 3-5 participants for the Phase 2 and 4 PPI groups and 8-10 participants for the Phase 5 cognitive interviews, reflecting current research practice $(25,26)$.

\section{Data Collection and Analysis}

An overview of the five phases of scale development is shown in Fig. 1.

\section{Phase 1: Secondary analysis}

Using the computer software program NVivo 12, a thematic secondary qualitative data analysis was undertaken by author O.K. retrospectively from 61 audio-recorded interview transcripts from four existing data sets $(21-24)$. Through three codeing phases, codes were generated which identified six broad key domains: pain, fatigue, physical consequence of disease, mood, social impact and healthcare related issues. Relevant accounts from the interview transcripts were then linked to the six domains, reviewed in detail and further refined looking specifically for DSD, with authors H.L. and J.S. Data that included distress was then flagged and grouped into a common 'theme' (see Figs. 1 and 2).

\section{Phase 2: Validation of Secondary Analysis}

A PPI group with patients diagnosed with RA $(n=4)$ was established to confirm whether the findings of the thematic analysis resonated with patients' experiences of living with rheumatological conditions. The 
PPI group deliberations were audio-recorded and summarized by O.K.

\section{Phase 3: Initial Item Generation}

Items for the Rheumatoid Arthritis Distress Scale (RADS) were generated from statements based on the anonymised transcripts used in the secondary data analysis, and the five themes of distress identified from that analysis (see Fig. 2). The initial list of items generated was refined by L.S. and H.L. Item order was considered at this stage by reviewing the literature $(27,28)$. At the end of Phase 3,44 items formed the first draft of the RADS.

\section{Phase 4: PPI Group}

A group of patients with RA $(n=3)$ were invited and took part in a PPI group. The 44-item first draft of the RADS was presented to participants, and questions about the content, phrasing, structure and layout of the PROM were explored. Participants were presented with alternative versions of the RADS to assess their preferences for scoring (numbers versus words). Field notes were taken from the PPI group deliberations, which were audio recorded and transcribed verbatim by L.S.

The same participants were contacted one week later via email to confirm changes made to the PROM (29). At the end of Phase 4, a 35-item RADS was drafted with three different layout versions.

\section{Phase 5: Cognitive Interviews}

Individual face-to-face $(n=4)$ or telephone $(n=5)$ cognitive interviews were conducted with participants with RA, by L.S., to identify any difficulties in understanding PROM instructions/content or scoring. Participants were asked to 'think aloud' as they read each item about what they understood it to mean, and if they thought the item was relevant $(30,31)$. Participants were asked to clarify how they 'came up' with their answer, to assess scoring discrimination (32). These cognitive interview techniques have been described elsewhere and used in PROM development (30-32).

By Phase 5, three versions of the RADS had emerged; one scored by word phrases one by numbers and one had supplementary condition-related contextual questions about time since diagnosis and level of disease activity. The versions were alternated during cognitive interviews after every second participant, to assess for preference to score using numbers versus words.

At the end of each interview, participants were asked which version they preferred, and if they thought the supplementary questions were relevant. Field notes were taken for the interviews, which were audio recorded, and transcribed verbatim by L.S. (interviews 1-2) and professional transcriber (interviews 3-9). To avoid participant burden, these transcripts were not sent for checking (33).

Data saturation (25) was continuously assessed by L.S. through monitoring the quality and level of new information emerging from subsequent interviews.

Phase 5 transcripts were uploaded to NVivo 12. Codes were created for each item (1 to 35). Sub-codes within these were generated to group the qualitative data into 'Discard', 'Keep', 'Rephrase' and 'Unsure'. 
Additional main codes created are as follows; 'Scoring' to analyse scoring preferences; 'Supplementary Questions' to analyse preferences on the additional questions; 'Instructions Clarity' to analyse if participants reported instructions were clear or not; 'Instructions Time Recall' to analyse preferences for the time recall period of the scale; 'Concept of DSD' to identify if participants validated the concept of DSD in RA. Data was coded by L.S. and reviewed by H.L.

Modifications to the RADS were finalised based on the data analysis of Phase 5.

\section{Psychometrician Consultation}

An academic psychometrician was consulted twice at the University to advise on the study design, scoring, numbering of items and modifications to the RADS, and recommendations for the future steps in the PROM development.

\section{Results}

Phase 1

Figure 2 shows the five themes of rheumatological disease distress formed from the secondary data analysis. Appendix 1 shows examples of relevant accounts from original transcripts illustrating identified themes of distress.

\section{Phase 2}

Overall, participants in the PPI group agreed that the themes of distress resonated with their experiences of living with RA (see Appendix 2).

\section{Phase 3}

Initial item generation yielded 94 items, categorised in order of the five themes of distress. After removal of repetitive statements, 44 items formed the first draft of the RADS. Items remained grouped in the themes of distress, but those relating to emotional distress were placed before items of physical distress to reflect the journey of patients with RA, following diagnosis, acceptance and impact of the disease. No other item order changes were made, informed by a literature review that indicated that there was no consensus for ordering of items $(27,28)$.

Phase 4

Appendix 3 shows examples of relevant accounts from Phase 4 PPI group. Participants unanimously reported that the themes and concept of DSD in RA resonated with their experiences of living with the disease. An additional theme of 'Work-related distress' was identified, added as a sub-theme of Social Distress (See Figure. 2). A corresponding new item to this was suggested, as was a new item regarding infections. 
PPI group feedback was to use word-based scoring and reduce the recall period in the PROM instructions from two months to two weeks, as a shorter duration was seen as the most reasonable amount of time one could remember accurately for scoring. Three additional supplementary questions based on time since diagnosis and disease activity were suggested, considered as contextually important for interpreting the distress score.

After the PPI group, a total of two new items had been created, 11 had been removed, and 23 had been rephrased.

By the end of Phase 4, three versions of the RADS existed, each with the same 35 items, but differed based on word or number scoring, and the presence of supplementary questions.

\section{Phase 5}

Thirteen members of a national RA charity members expressed interest with nine participating. Four could not take part due to scheduling conflicts.

Phase 5 interview data that was uploaded onto NVivo 12 yielded the results presented below. See Appendix 4 for relevant accounts from interview transcripts

\section{(i) The Concept of DSD in RA}

Five participants clearly validated DSD as an entity in RA and three inferred it was a valid concept when discussing certain items, for example in relation to pain.

One participant did not seem to have experienced DSD but verbalised she could see how the items were relevant and distressing for others living with RA.

\section{(ii) Item Relevancy and Rephrasing}

Participants $(n=9)$ reported that most of the items were relevant to their personal experiences living with RA or would be to others living with RA. Participants suggested minor rephrasing of 19 items.

\section{(iii) Item Creation}

New items were suggested in relation to the theme of treatment-related, physical-related, and healthcarerelated distress $(n=5)$.

\section{(iv) Item Removal or Combination}

Five participants reported that two items regarding RA pain were similar and could be combined, and that the word 'distress' better explained feelings towards their pain than 'anger'. Two disagreed.

Seven participants reported that two items concerning test results and healthcare professionals' explanations of treatments were similar and could be combined. 
(v) Item Order

Participants had few strong opinions on the order of items on the PROM and stated that the current sequence made sense. However, six suggested that item 'I feel overwhelmed living with RA' could move to the end of the scale.

\section{(vi) Supplementary Questions}

Nine, seven and six participants preferred to keep supplementary questions 1, 2 and 3 in the scale, respectively. Participants reported that the questions gave context to scoring the PROM.

\section{(vii) PROM Scoring}

Six participants reported preferring to answer the PROM using word scoring, two preferred numbers, and one held no preference.

Those who preferred word labels reported being able to 'relate' more to words and that it helped them consider each item more carefully than if scoring on a numerical scale.

\section{(viii) PROM Instructions}

Overall $(n=9)$, participants reported that they understood PROM instructions and they were clear. Regarding the timeframe mentioned in the instructions, they reported that due to the variability of their condition, two weeks may not accurately capture distress levels.

Some participants $(n=3)$ suggested to extend the timeframe to one month, some $(n=1)$ to three months, some $(n=2)$ were unsure, while others $(n=3)$ agreed with two weeks.

\section{Demographic Data}

Although demographic data was not formally collected for Phases 2, 4 and 5 participants, some information unintentionally was disclosed; Most participants were female, Caucasian, and revealed their occupations were in the banking, business, or healthcare-sectors. Disease duration varied from two to 40 years in cognitive interview participants.

\section{Psychometrician Consultation}

The psychometrician validated the study design and confirmed word-based scoring to be appropriate for a PROM so long as presented in ordinal format for summation of scoring. The psychometrician also confirmed the supplementary questions as relevant and suggested these to be inserted at the end of the PROM to avoid potentially influencing participants scoring. Finally, the psychometrician suggested consideration of the time recall period in the instructions to ensure consistency and validity of participant answers.

\section{Final Changes to the RADS}


Final changes were made to the RADS based on the results and analysis from Phase 5 data presented above (See RADS Final Version in Appendix 5).

A preliminary decision was made for instructions to score based on the previous three months rather than two weeks, ensuring enough timeframe to capture different aspects of distress. Word-based scoring was chosen as it was the predominant preference from Phase 4 and 5 participants. No major differences were noted from gross observation of scoring patterns when alternating the different versions in Phase 5.

In total, 19 items on the PROM were rephrased, two were removed, and six new items were created. The three supplementary questions were retained in response to the participants' preference, and the psychometrician's advice.

For item order, new items were inserted into the PROM alongside those from their corresponding theme. Item 'I feel overwhelmed living with RA' was moved to the end to become item 39 on the RADS as per the participants' $(n=6)$ suggestions. Therefore, after analysis of the data in Phase 5 of the RADS development, the final version consisted of a 39-item scale with three supplementary questions (See Appendix 5).

\section{Discussion}

\section{Presentation of Principle Findings}

This study described the five initial phases of development of the RADS, a PROM to identify DSD in people with RA. The PPI group and cognitive interviews confirmed empirically the findings from the secondary data analysis that RA distress does exist as an important entity and appears distinct from other conditions like clinical depression. The 39-item RADS has face and content validity with people with RA. Conceptually, the 39 items link to one of five themes of RA distress. The RADS is now available for further psychometric evaluation in clinical and research populations.

\section{Comparisons of evidence with the wider literature}

The themes of RA distress (Fig. 2) from this study are similar to those identified in type 1 and 2 diabetes $(18,34), \operatorname{IBD}(9)$ and to a lesser extent cancer distress scales (35). These long-term diseases share constructs of emotional, healthcare-related, treatment-related and social distress. Emotional distress items from two validated diabetes distress measures $(18,34)$ echo the RADS, describing difficulties of illness acceptance and worries about future complications. In this study the predominant emotion for people with RA seemed to be of frustration, although minor themes of anger also emerged, with some participants reporting that anger was not an emotion they were 'allowed' to feel or express, resonating findings from previous qualitative studies (36).

Cancer (35), diabetes $(18,34)$, IBD (17) and RA scales have all included items about healthcare-related distress. In diabetes and IBD 'concerns not taken seriously' by healthcare professionals emerged, while in RA lack of clinicians' time spent to assess the condition and provide emotional support caused distress. 
In both cancer and RA, distress over lack of adequate information from healthcare professionals has been described (35).

Treatment-related distress in IBD and RA included concerns over side effects, while in diabetes lack of confidence/motivation in self-management, and guilt about failing with treatment regimens, seems predominant. Social-related distress common to all four long-term conditions highlighted a lack of understanding from others about their illnesses.

People with RA, IBD or cancer, but not diabetes, demonstrate symptom-related distress. People with RA are distressed about their joint pain, stiffness, and fatigue. Pain may explain the difference in degree of depression between RA and healthy controls (37) and there is some evidence that psychological distress in RA can be secondary to pain rather than vice versa (38). The link between pain and DSD in RA, however, has not been previously investigated.

Elevated diabetes distress is prevalent in $20-40 \%$ of people with type $1(39,40)$ and in $36 \%$ of people with type 2 (41) diabetes. Four systematic reviews have explored psychological interventions in diabetes. In general, reviews found psycho-educational treatments resulted in a low to moderate effect on DSD reduction and more intensive and longer duration interventions seem to achieve a greater effect $(13,14$, $42,43)$. One systematic review demonstrated that motivational interviewing significantly reduced diabetes distress and improved glycaemic control (13). Furthermore, diabetes-tailored interventions, as opposed to general mindfulness interventions, showed to most likely improve both DSD and glycaemic control in people with type 1 and 2 diabetes (14).

In a cross-sectional study of 189 cancer patients, 58\% demonstrated elevated DSD (6). Psychosocial interventions may improve quality of life and alleviate distress in people with cancer, although systematic reviews have not focussed on DSD as a primary outcome $(44,45)$.

In IBD and RA, the prevalence of DSD is not yet known, nor what effective treatment may entail. However, psychological interventions can result in small to moderate improvements in biopsychosocial outcomes for patients with RA (46).

\section{Strengths and limitations of the study}

The strengths of this research are its systematic study design, which was validated by a psychometrician, and followed recommended guidelines of PROM development (19) and reporting (20). The secondary data analysis was an ethically efficient way to make good use of anonymised data. People with RA were involved throughout the process of development. Changes to the PROM after Phase 4 were endorsed by PPI group participants, ensuring accurate interpretation of data (29). The robustness and validity of data was further strengthened by inclusion of diverse cases/minor themes (20). One notable strength of our demographics was the mixture of early and established RA.

The PPI groups in this study had taken part in several similar research projects in the past and embraced the topic of RA distress. The data generated therefore led to high 'information power' (47). The final two 
cognitive interviews in Phase 5 did not yield as much 'new information' indicating close 'data saturation' (25).

This research has limitations. Our study would have benefited from including more males and people from diverse socioeconomic and ethnic backgrounds. RA incidence can be 4-5 times higher in females (48), hence the difficulty in recruiting males for this study. Cognitive interview participants were recruited from one national charity, which could explain the homogeneity of this sample. The team did not send participants transcripts for fact/content checking to minimise research burden and/or fatigue (33). Finally, participants in Phase 1 were not specifically asked about RA distress and since the 39 items were generated during secondary analysis of this data, some items areas may be missing from the RADS.

\section{Recommendations for practice, policy and research}

DSD in RA appears to be a new important concept and its identification comes at a pivotal time; the 2018 updated NICE guidelines for RA management in adults recommended clinicians to assess the effect RA has on the patients' life (49). Patients with RA report that the most frequent reasons for flare up of joint symptoms is psychological stress (50). It seems important, therefore, to have a means to identify this. The RADS is a PROM that holds the potential to be important in clinical practice and research in the future.

There is a need to establish face and content validity of the RADS in a more diverse patient population. Item order could be investigated for reliability differences between item grouping or intermixing. Currently no gold standard for RA distress exists. Therefore, the RADS could be evaluated against modified validated diabetes and/or IBD distress scales, considering the similarities in distress domains.

Following detailed psychometric evaluation, the RADS has the potential to be employed in larger longitudinal studies to identify the prevalence of RA distress. It would be worth investigating whether DSD is associated with clinical outcomes in RA. Effective psychological interventions for DSD, as implemented in type 1 and 2 diabetes patients (13), would be welcome by people with RA and their carers.

\section{Conclusions}

In summary, this study offers evidence for DSD as an important entity experienced by many with a variety of long-term conditions, including RA, with some shared domains of distress and some unique aspects to each condition. The 39-item RADS has acceptable face and content validity in people with RA. RA distress appears to be distinct from clinical depression or anxiety disorders. The RADS has the potential to be a useful tool for identifying RA distress.

\section{Abbreviations}

Rheumatoid Arthritis - RA

Disease-specific distress - DSD 
Inflammatory Bowel Disease - IBD

Patient Reported Outcome Measure - PROM

Antiphospholipid Syndrome - APS Idiopathic Inflammatory Myopathy - IIM Patient and Public Involvement - PPI Rheumatoid Arthritis Distress Scale - RADS

\section{Declarations}

\section{Ethics approval and consent to participate}

Please see Methods section. The local University Ethics Committee provided ethics approval for Phases 3-5 on 24.04.2018 (REC Number: MRS-17/18-6443). Participants undertook informed consent procedures

\section{Consent for Publication}

Not applicable

\section{Availability of data and materials}

The datasets used and/or analysed during the current study are available from the corresponding author on reasonable request.

\section{Competing interests}

JS received royalties from the University of Warwick in relation to the Diabetes Manual programme There are no conflicts of interest for all other authors.

\section{Funding}

This work was supported by King's College London and submitted in partial fulfilment for the MSc Degree in Advanced Neuro-musculoskeletal Physiotherapy.

\section{Authors' contributions}

L.S: substantial contribution to acquisition, analysis and interpretation of data, drafting and revising the article, and approving the final submitted version

O.K: substantial contribution to acquisition, analysis and interpretation of data, and approving the final submitted article

J.S.: substantial contribution to study design and conception, analysis and interpretation of data, drafting and revising the article, and approving the final submitted version 
H.L.: substantial contribution to study design and conception, analysis and interpretation of data, drafting and revising the article, and approving the final submitted version

\section{Acknowledgements}

I would like to thank all of the people from the focus group and interviews who contributed to the development of the RADS for their expertise, time and enthusiasm. In addition, I am grateful to Dr. Silia Vitoratu for her sound psychometric advice

\section{References}

1. Scott DL, Wolfe F, Huizinga TWJ. Rheumatoid Arthritis Lancet. 2010;376:1094-108.

2. Shih M, Hootman JM, Strine TW, Chapman DP, Brady TJ. Serious psychological distress in U.S. adults with arthritis. J Gen Intern Med. 2006;21(11):1160-6.

3. Matcham F, Rayner L, Steer S, Hotopf M. The prevalence of depression in rheumatoid arthritis: a systematic review and meta-analysis. Rheumatology. 2013;52(12):2136-48.

4. el-Miedany $\mathrm{YM}$, el-Rasheed $\mathrm{AH}$. Is anxiety a more common disorder than depression in rheumatoid arthritis? Joint Bone Spine. 2002;69(3):300-6.

5. Herschbach P, Keller M, Knight L, Brandl T, Huber B, Henrich G, et al. Psychological problems of cancer patients: a cancer distress screening with a cancer-specific questionnaire. $\mathrm{Br} \mathrm{J}$ Cancer. 2004;91(3):504-11.

6. Haun MW, Sklenarova H, Brechtel A, Herzog W, Hartmann M. Distress in cancer patients and their caregivers and association with the caregivers' perception of dyadic communication. Oncol Res Treat. 2014;37(7-8):384-8.

7. Book K, Marten-Mittag B, Henrich G, Dinkel A, Scheddel P, Sehlen S, et al. Distress screening in oncology-evaluation of the Questionnaire on Distress in Cancer Patients-short form (QSC-R10) in a German sample. Psychooncology. 2011;20(3):287-93.

8. Dennick K, Sturt J, Hessler D, Purssell E, Hunter B, Oliver J, et al. High rates of elevated diabetes distress in research populations: A systematic review and meta-analysis. International Diabetes Nursing. 2015;12(3):93-107.

9. Woodward S, Dibley L, Coombes S, Bellamy A, Clark C, Czuber-Dochan W, et al. Identifying diseasespecific distress in patients with inflammatory bowel disease. Br J Nurs. 2016;25(12):649-60.

10. Association AP. Diagnostic and statistical manual of mental disorders: DSM-5. Arlington: American Psychiatric Association; 2013.

11. Fisher L, Skaff MM, Mullan JT, Arean P, Mohr D, Masharani U, et al. Clinical depression versus distress among patients with type 2 diabetes: not just a question of semantics. Diabetes Care. 2007;30(3):542-8.

12. Fisher L, Mullan JT, Arean P, Glasgow RE, Hessler D, Masharani U. Diabetes distress but not clinical depression or depressive symptoms is associated with glycemic control in both cross-sectional and 
longitudinal analyses. Diabetes Care. 2010;33(1):23-8.

13. Sturt J, Dennick K, Hessler D, Hunter BM, Oliver J, Fisher L. Effective interventions for reducing diabetes distress: systematic review and meta-analysis. International Diabetes Nursing. 2015;12:4055 .

14. Schmidt CB, van Loon BJP, Vergouwen ACM, Snoek FJ, Honig A. Systematic review and metaanalysis of psychological interventions in people with diabetes and elevated diabetes-distress. Diabet Med. 2018;35(9):1157-72.

15. Hofmann D, Ibrahim F, Rose D, Scott DL, Cope A, Wykes T, et al. Expectations of new treatment in rheumatoid arthritis: developing a patient-generated questionnaire. Health Expect. 2015;18(5):9951008.

16. Yazdany J, Trupin L, Gansky SA, Dall'Era M, Yelin EH, Criswell LA, et al. Brief index of lupus damage: A patient-reported measure of damage in systemic lupus erythematosus. Arthritis Care Res. 2011;63(8):1170-7.

17. Dibley L, Czuber-Dochan W, Woodward S, Wade T, Bassett P, Sturt J, et al. Development and Psychometric Properties of the Inflammatory Bowel Disease Distress Scale (IBD-DS): A New Tool to Measure Disease-Specific Distress. Inflamm Bowel Dis. 2018.

18. Polonsky WH, Fisher L, Earles J, Dudl RJ, Lees J, Mullan J, et al. Assessing psychosocial distress in diabetes: development of the diabetes distress scale. Diabetes Care. 2005;28(3):626-31.

19. Rothrock N, Kaiser K, Cella D. Developing a Valid Patient-Reported Outcome Measure. Clin Pharmacol Ther. 2011;90(5):737-42.

20. Tong A, Sainsbury P, Craig J. Consolidated criteria for reporting qualitative research (COREQ): a 32item checklist for interviews and focus groups. Int J Qual Health Care. 2007;19(6):349-57.

21. Prothero L, Georgopoulou S, Galloway J, Williams R, Bosworth A, Lempp H. Patients' and carers' views and expectations about intensive management for moderate rheumatoid arthritis: a qualitative study. Psychol Health Med. 2016;21(8):918-25.

22. Pollard LC, Graves H, Scott DL, Kingsley GH, Lempp H. Perceived barriers to integrated care in rheumatoid arthritis: views of recipients and providers of care in an inner-city setting. BMC Musculoskelet Disord. 2011;12:19.

23. Campbell R, Hofmann D, Hatch S, Gordon P, Lempp H. Muscle disorders111. The impact of fatigue in patients with idiopathic inflammatory myopathy: a mixed method study. Rheumatology. 2012;51(suppl_3):iii93-139.

24. Bearne L, D'Cruz D, Nel L, Georgopoulou S, Andrews J, Bieles J, et al. 236 The prevalence and impact of fatigue and physical activity in people with primary antiphospholipid syndrome: a mixed methods study. Rheumatology. 2019;58.

25. Carlsen B, Glenton C. What about N? A methodological study of sample-size reporting in focus group studies. BMC Med Res Methodol. 2011;11(26):1-10.

26. Peterson C, Peterson N, Powell K. Cognitive Interviewing for Item Development: Validity Evidence Based on Content and Response Processes. Meas Eval Couns Dev. 2017;50(4):217-23. 
27. Rattray J, Jones MC. Essential elements of questionnaire design and development. J Clin Nurs. 2007;16(2):234-43.

28. Cheung Y-B, Wong L-C, Tay M-H, Toh C-K, Koo W-H, Epstein R, et al. Order effects in the assessment of quality of life in cancer patients. Qual Life Res. 2004;13(7):1217-23.

29. Thomas D. Feedback from research participants: are member checks useful in qualitative research? Qual Res Psychol. 2017;14(1):23-41.

30. Ahmed N, Bestall JC, Payne SA, Noble B, Ahmedzai SH. The use of cognitive interviewing methodology in the design and testing of a screening tool for supportive and palliative care needs. Support Care Cancer. 2009;17(6):665-73.

31. Drennan J. Cognitive interviewing: verbal data in the design and pretesting of questionnaires. J Adv Nurs. 2003;42(1):57-63.

32. Beatty P, Willis G. Research Synthesis: The Practice of Cognitive Interviewing. Public Opin Q. 2007;71(2):287-311.

33. Mero-Jaffe I. 'Is that what I said?' Interview Transcript Approval by Participants: An Aspect of Ethics in Qualitative Research. Int J Qual Methods. 2011;10(3):231-47.

34. Polonsky WH, Anderson BJ, Lohrer PA, Welch G, Jacobson AM, Aponte JE, et al. Assessment of diabetes-related distress. Diabetes Care. 1995;18(6):754-60.

35. Herschbach P, Marten-Mittag B, Henrich G. Revision und psychometrische Prufung des Fragebogens zur Belastung von Krebskranken (FBK-R23). 2003;12:69-76.

36. Zangi $\mathrm{H}$, Hauge M, Steen E, Finset A, Hagen K. 'I am not only a disease, I am so much more'. Patients with rheumatic diseases' experiences of an emotion-focused group intervention. Patient Educ Couns. 2011;85:419-24.

37. Dickens C, McGowan L, Clark-Carter D, Creed F. Depression in rheumatoid arthritis: a systematic review of the literature with meta-analysis. Psychosom Med. 2002;64(1):52-60.

38. Smedstad LM, Vaglum P, Kvien TK, Moum T. The relationship between self-reported pain and sociodemographic variables, anxiety, and depressive symptoms in rheumatoid arthritis. J Rheumatol. 1995;22(3):514-20.

39. Sturt J, Dennick K, Due-Christensen M, McCarthy K. The detection and management of diabetes distress in people with type 1 diabetes. Curr Diab Rep. 2015;15(11):101.

40. Fisher L, Polonsky WH, Hessler DM, Masharani U, Blumer I, Peters AL, et al. Understanding the sources of diabetes distress in adults with type 1 diabetes. $J$ Diabetes Complications. 2015;29(4):572-7.

41. Perrin NE, Davies MJ, Robertson N, Snoek FJ, Khunti K. The prevalence of diabetes-specific emotional distress in people with Type 2 diabetes: a systematic review and meta-analysis. Diabet Med. 2017;34(11):1508-20.

42. Chew BH, Vos RC, Metzendorf MI, Scholten R, Rutten G. Psychological interventions for diabetesrelated distress in adults with type 2 diabetes mellitus. Cochrane Database of Systematic Reviews. 
2017(9).

43. Mathiesen AS, Egerod I, Jensen T, Kaldan G, Langberg H, Thomsen T. Psychosocial interventions for reducing diabetes distress in vulnerable people with type 2 diabetes mellitus: a systematic review and meta-analysis. Diabetes Metab Syndr Obes. 2019;12:19-33.

44. Teo I, Krishnan A, Lee GL. Psychosocial interventions for advanced cancer patients: A systematic review. Psycho-Oncology. 2019;28(7):1394-407.

45. Raingruber $B$. The effectiveness of psychosocial interventions with cancer patients: an integrative review of the literature (2006-2011). ISRN Nurs; 2011.

46. Prothero L, Barley E, Galloway J, Georgopoulou S, Sturt J. The evidence base for psychological interventions for rheumatoid arthritis: A systematic review of reviews. Int J Nurs Stud. 2018;82:20-9.

47. Malterud K, Siersma VD, Guassora AD. Sample Size in Qualitative Interview Studies: Guided by Information Power. Qual Health Res. 2015.

48. Kvien TK, Uhlig T, Odegard S, Heiberg MS. Epidemiological aspects of rheumatoid arthritis: the sex ratio. Ann N Y Acad Sci. 2006;1069:212-22.

49. National Institute for Health and Care Excellence. (2018). Rheumatoid arthritis in adults: management NICE guidelines (NG100) [Available from: https://www.nice.org.uk/guidance/ng100.

50. Yilmaz V, Umay E, Gundogdu I, Karaahmet ZO, Ozturk AE. Rheumatoid Arthritis: Are psychological factors effective in disease flare? Eur J Rheumatol. 2017;4(2):127-32.

\section{Figures}




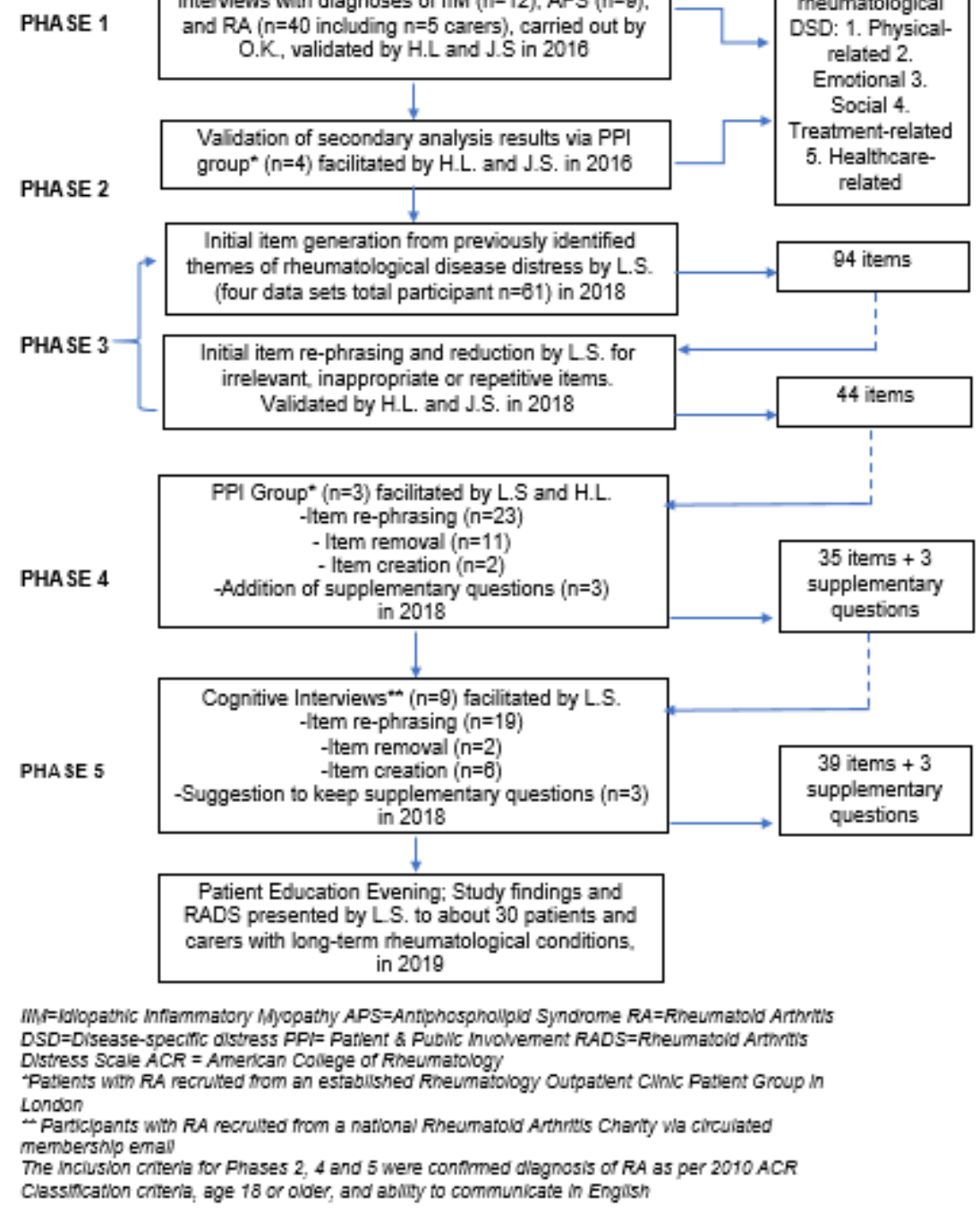

\section{Figure 1}

Flow chart of phases of development of the RADS 


\section{Physical related distress}

a. Pain (7)

b. Fatigue (15)

c. Physical consequence of disease (21)

\section{Emotional distress}

a. Acceptance and burden of disease (11)

b. Worry about prognosis (15)

\section{Social Distress}

a. Impact on personal relationships (8)

b. Work-related distress

\section{Treatment related distress (12)}

5. Health care related distress (16)

In brackets () is the frequency of relevant accounts of DSD that were identified for each corresponding theme in Phase 1

Underlined is a new sub-theme that emerged from Phase 4 of the study

\section{Figure 2}

Themes of Rheumatological Disease Distress

\section{Supplementary Files}

This is a list of supplementary files associated with this preprint. Click to download.

- APPENDIX1.docx

- APPENDIX2.docx

- APPENDIX3.docx

- APPENDIX4.docx

- APPENDIX5.docx 